\title{
Collecting Duct Carcinoma
}

National Cancer Institute

\section{Source}

National Cancer Institute. Collecting Duct Carcinoma. NCI Thesaurus. Code C6194.

Also known as collecting duct carcinoma, this is a rare type of renal carcinoma. It arises from the collecting ducts of the renal medulla, and most authors suggest that this is an aggressive tumor. 